\title{
RESPONSIVENESS TO HCG IN POSTMENOPAUSAL WOMEN WITH ADRENAL ADENOMAS
}

B. Popovic, Dj. Macut, I. Bozic, T. Bogavac, D. llic, T. Isailovic, V. Elezovic, S. Ognjanovic, S. Damjanovic Clinic for Endocrinology, Diabetes and Metabolic Diseases, Clinical Centre of Serbia, Belgrade, Serbia

Introduction: Luteinizning hormone (LH/hCG) responsiveness has been documented in both normal and pathological adrenal glands, but it is unclear whether chronic exposure to LH could affect adrenal function by increasing LH receptor (LHR) expression.

Subjects and methods: We studied 12 postmenopausal women with previously documented adrenal tumor. All patients underwent standard hormonal testing for assessment of tumor functionality. Then hCG test was performed with measurement of adrenal androgens and estradiol levels from the blood. A $50 \%$ or more change of plasma level from baseline was considered as positive response. Statistical analysis was done by SPSS software.

Results: Our patients were 60.4 5.8 years old (54-71), and were 12.47 .9 years postmenopausal (1-23). Five patients $(41.7 \%)$ had Cushing's syndrome, 3 of which had bilateral adrenal tumors, and the remaining 7 had unilateral, clinically nonfunctional adrenal adenoma. By adequate rise in levels of estradiol, testosterone, DHEA-S and 17-OH progesterone we identified 6 patients (50\%) that could be categorized as responders to hCG.
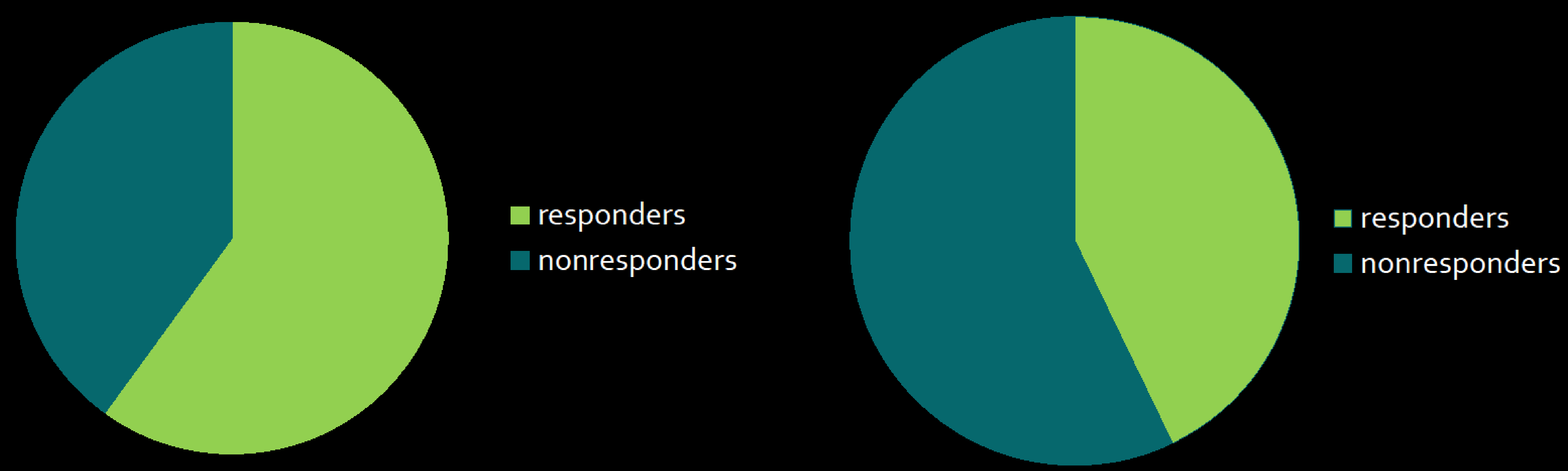

Three out of 5 patients with Cushing's syndrome were responders, and 3 out of 7 patients with nonfunctional tumors. There was no significant correlation between $\mathrm{LH}$ level and years after menopause with percent of change in any hormone tested $(p>0.05)$.

Fig 1: Patients with Cushing's

Fig 2: Patients with nonfunctional adenoma

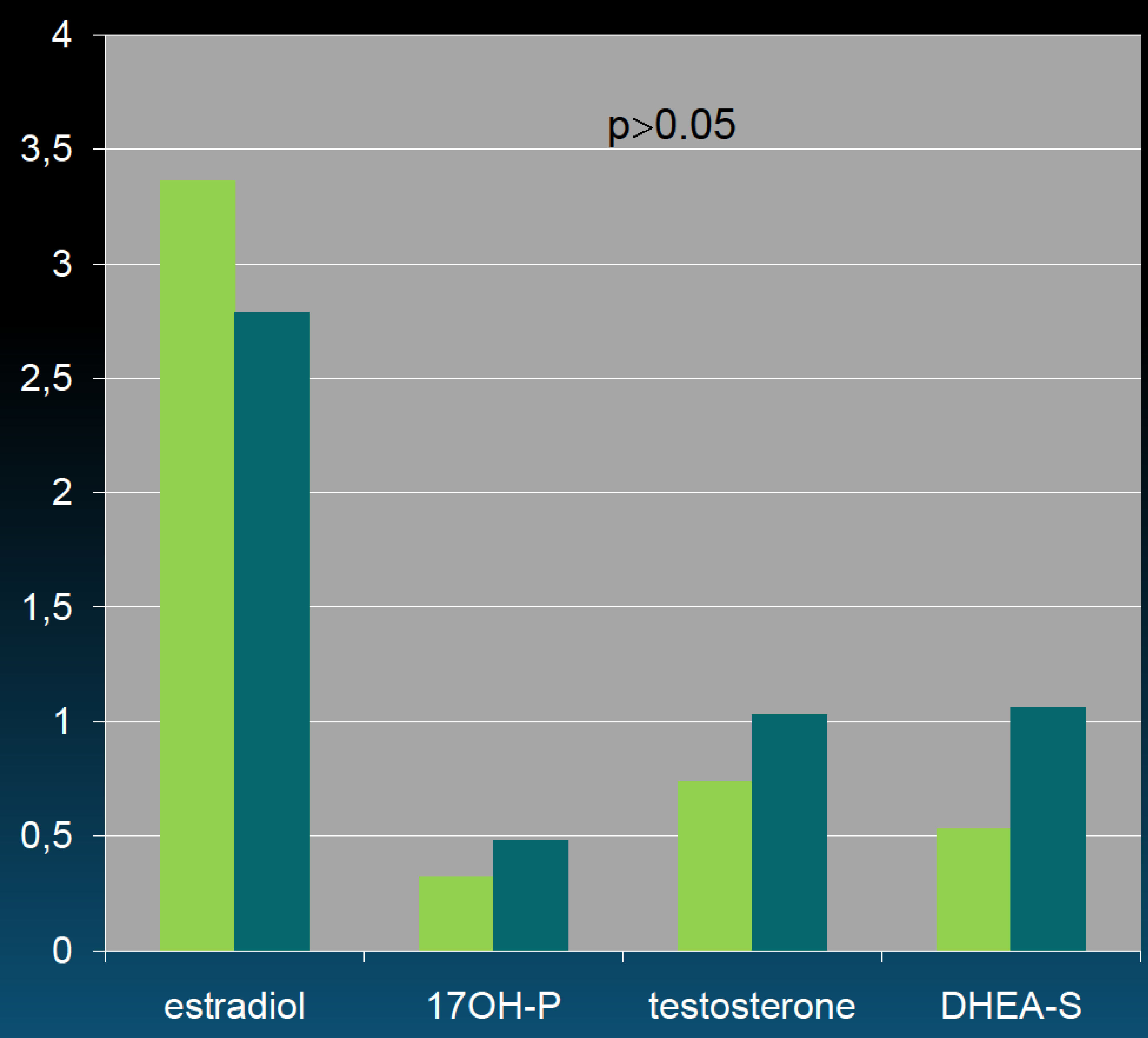

Fig 3: Differences in basal hormone values

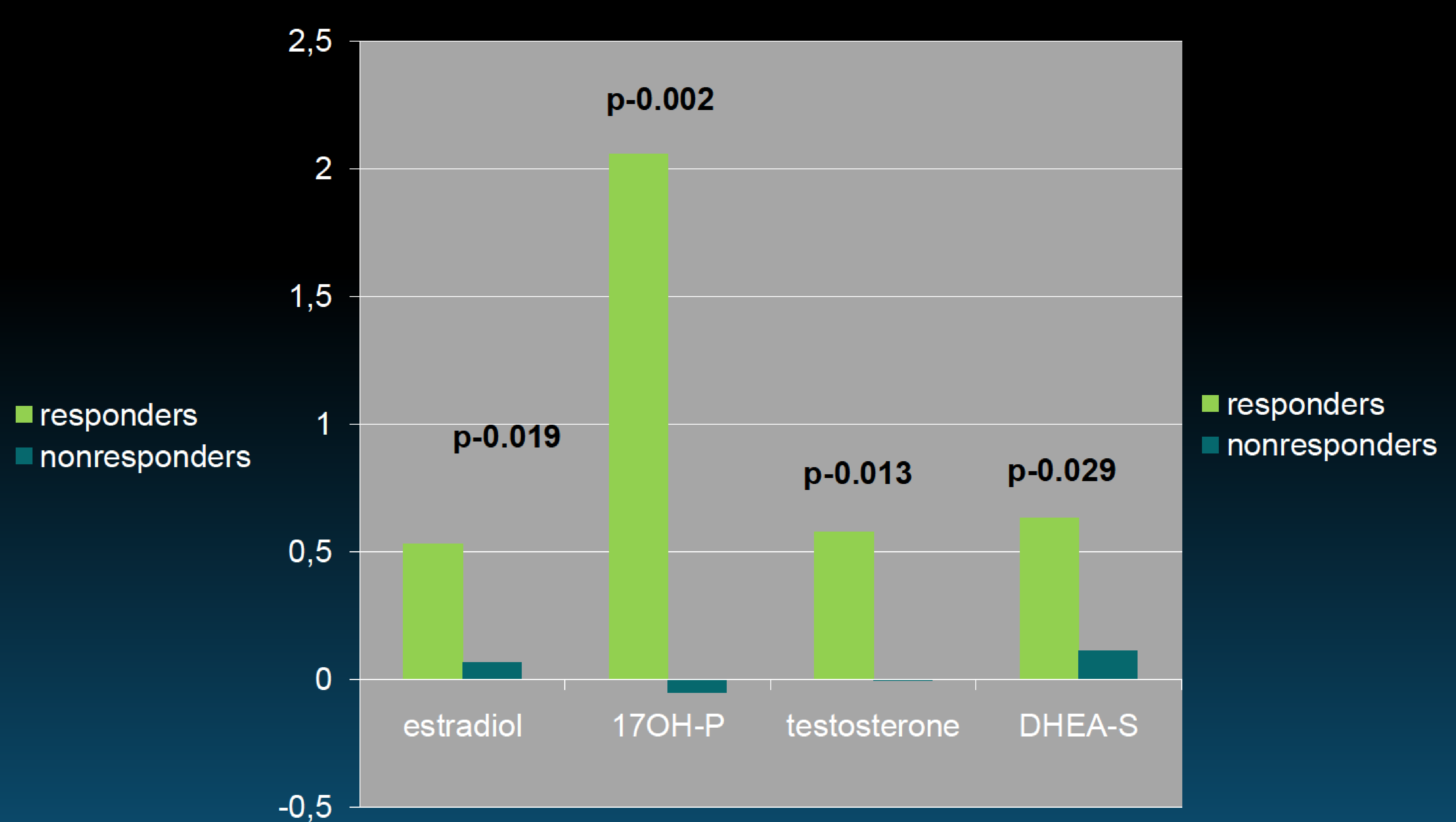

Fig 4: Differences in percents of hormone level change

Responders and non-responders were not significantly different in any of the basal hormonal levels ( $p>0.05)$, but were significantly different in percents of hormone level change of all tested hormones (Figures 3 and 4). Among responders, there was no difference in hormonal responses to hCG regarding presence of Cushing's syndrome ( $p>0.05$ for all).

Conclusion: Responsiveness to hCG was more prevalent in our group of postmenopausal women than previously reported in patients with adrenal adenomas, suggesting a possible role of long term LH exposure.

\begin{tabular}{|c|c|c|c|c|c|}
\hline 올 20--GP & Adrenal 2 & Poster & ECE2016 & 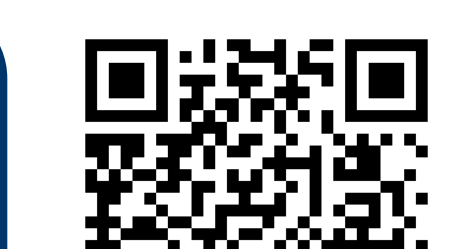 & Porter \\
\hline & Bojana Popovic & & 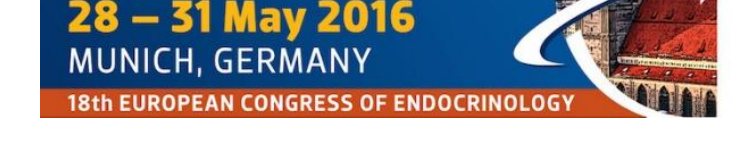 & 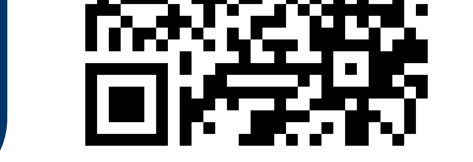 & Jess \\
\hline
\end{tabular}

